\title{
Towards a Modern, Integrated Virtual Laboratory System
}

\author{
Tamás Budai, Miklós Kuczmann
}

Department of Automation, Széchenyi István University

Egyetem tér 1, H-9026 Györ, Hungary

budai.tamas@sze.hu; kuczmann@sze.hu

\begin{abstract}
The aim of this paper is to give an overview on virtual and remote laboratory systems and to evaluate current solutions focusing on feasibility and applicability in higher education. Based on the conclusions of this evaluation, a new set of requirements are established against a modern virtual laboratory system. Finally, an overview of state of the art infocommunication technologies, including cognitive infocommunication are presented, which can help create high user experience in the new virtual laboratory environment.
\end{abstract}

Keywords: virtual; laboratory; system; overview; design

\section{Introduction}

In the past decade distance learning or e-Learning has matured and now a widely used form of higher education worldwide. Most of these systems only provide a platform to give access to static content like textbooks and other course materials. Nowadays remote and virtual laboratories are also widespread, especially in engineering disciplines [1] [2], where laboratory exercises are playing a key part, helping students to connect lexical knowledge to the real world. These systems provide interactive learning tools which can complement the lectures in the classroom.

In the first part, a short discussion on the benefits and drawbacks of virtual and remote laboratories is presented. The terms remote and virtual are often used inconsistently in literature, therefore a clarification on the definition of these two is necessary [3]. In the second part the overview of the current state of remote and virtual laboratories is presented and a set of example systems are then analyzed to identify key factors that must be taken into account when evaluating a virtual laboratory system. After the overview and evaluation is complete the results and experiences are summarized. Finally, based on these results and by utilizing state of the art infocommunication technologies, a possible new way is proposed on how to implement modern virtual laboratories. 


\section{Remote and Virtual and Laboratories}

The two common types of online laboratory systems are remote and virtual laboratories. From the perspective of the end-users, these look very similar, by providing an online service, which is available via either a website or a client application, but has a very important difference in their backend.

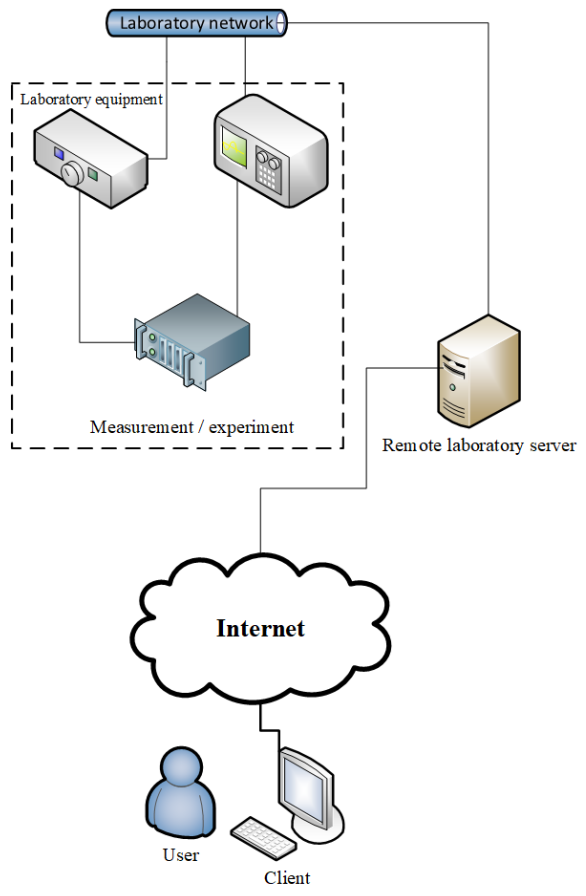

a,

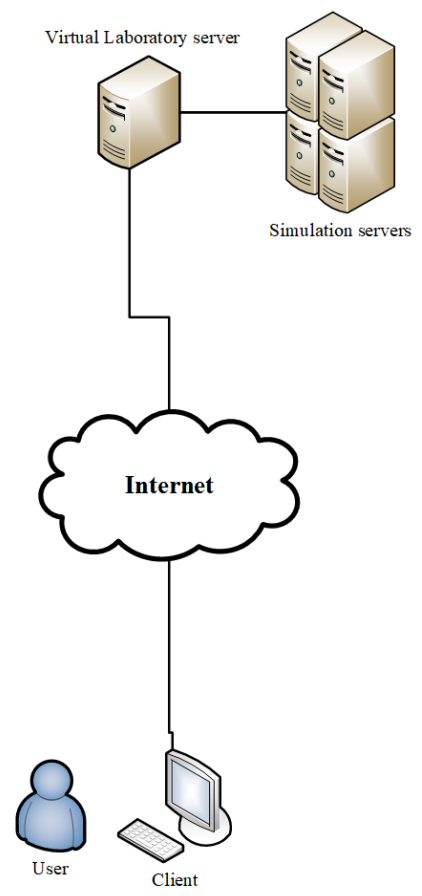

b,

Figure 1

Figure 1 shows the main difference between remote (Fig. 1a) and virtual (Fig. 1b) laboratories. In a remote laboratory all the hardware components (e.g. measurement equipment) physically exist somewhere in the institution. By connecting this equipment to the network, the relevant part of the experiment can be controlled and examined by students from a remote location. On the other hand, in a virtual laboratory all of the above components are entirely simulated by software and can be accessed remotely by users. Distinguishing by their purpose of use, remote laboratories are both used in collaborative research and education. Virtual laboratories on the other hand are mostly suitable for education. 
Beside these differences, the two have many common features, therefore for the first time comparison of remote and virtual laboratories will be treated as one category.

\subsection{Advantages of Remote and Virtual Laboratories}

From the perspective of the university and the professor, a virtual or remote laboratory provides the possibility to break out from the physical constraints of a traditional laboratory and make the exercises available to a wider audience of students. Because all of these labs involve infocommunication technology, it is possible to track student behavior and collect statistics, which can be used to generate useful feedback on the effectiveness of a given virtual or remote laboratory and can be used to pinpoint problems with a given material.

From the perspective of the students, the most appealing advantage is the ability to do the experiment in a self-paced manner and that the lab is accessible anytime from anywhere.

\subsection{Disadvantages of Remote and Virtual Laboratories}

One disadvantage of virtual and remote laboratories is the lack of hands-on contact with devices and equipment, therefore a virtual or remote laboratory does not improve manual skills i.e. dexterity, which is necessary to achieve the same experiment result when working with real-life equipment. This effect can be reduced to an extent by using high fidelity $3 \mathrm{D}$ models of the equipment.

Another disadvantage comes from the fact that the virtual environment is similar to video games. Students tend to look at the virtual experiment as a game, therefore they lose the sense of seriousness. In remote laboratories, students are unable to cause damage by making mistakes, because all controlled components have limits predefined and checked by the environment. These labs do not require the same level of discipline and caution that is necessary to do the same experiment in a traditional laboratory in a safe manner.

\subsection{Advantages of Virtual Laboratories}

A large number of laboratory experiments are either dangerous or involve expensive equipment, therefore a supervisor must be present in the laboratory and the necessary theoretical knowledge of the student must be tested before they can start the experiment. These two factors rule out the possibility to simultaneously do the experiment and gain theoretical knowledge in a learning-by-doing manner for the student. 
In a virtual laboratory, students can conduct experiments in a safe manner and the virtual laboratory can simulate extreme conditions, like altered gravity or very high ambient temperature, which would be impossible or very expensive to do in a traditional or remote laboratory.

The absence of actual equipment (hardware) means the number concurrent labs are only limited by system resources, the virtual hardware does not age nor wear out, which means lower operational costs. It is also cheaper to establish a new experiment, because there is no need to buy new equipment.

\subsection{Disadvantages of Virtual Laboratories}

The only real disadvantage of a virtual laboratory over a remote one comes from the fact that a remote lab is backed by real hardware and therefore it produces all the behavior of that hardware without the need to create complex mathematical models that can never take into account all details of a real, existing physical system. Fortunately, the impact of this disadvantage depends on the use case of the laboratory system. The precision that a mathematical model can achieve is, most of the time, adequate for the purpose of education and demonstration. However, it is important to keep in mind that the model and the simulation must be sophisticated enough to cover all the important aspects of the given experiment: it is important to correctly model the phenomena that is the subject of the given lab (e.g.: precision of a measurement equipment is affected by the ambient temperature).

\section{Overview and Evaluation}

\subsection{Current State of Virtual Laboratories}

Since virtual laboratories have numerous advantages over traditional and remote labs, virtual laboratory design and development became a widely researched topic in the past few years. Many research groups, universities and even members of the industry developed their own virtual laboratories, covering a very wide area of disciplines [4] [5] [6]. In current scientific literature, two major categories of virtual laboratories can be distinguished.

In the first category [4] [7] Error! Reference source not found.[8], there is virtual laboratory implementations that were designed and suitable for a single specific purpose, i.e. for a single experiment. These implementations do not employ systematic approaches on design, e.g. does not take into account the re- 
usability or different components. Instead, the focus is on the experiment itself and the software components that are already playing a role in this experiment is also used to implement the necessary services for the remote laboratory, e.g. the webserver. Most of the time, this approach yields quick results because the teacher or researcher are already familiar with the software, but in the long run it is a source of multiple problems. First, the software, which is perfectly suitable for the experiment, e.g. LabVIEW or Matlab may contain a module to implement a webserver. However, these were not designed to handle the challenges, e.g. a large number concurrent requests that webserver software was designed for. Second, it is impossible to integrate these components with external services, like authentication and authorization, accounting, and data storage. Third, it is very hard to create a consistent user interface for the end-users of the system.

In the second category [9] [10], there are proposed virtual laboratory system designs, some of them with reference implementations, which were created by following a systematic design approach, employing design patterns and best practices from the field of web-development. This approach successfully eliminates the problems mentioned in the first category. However, it poses a new requirement against the teams who want implement a new experiment, namely that they have to learn the usage of new tools that are specific to webdevelopment. In engineering disciplines, this is often not a problem because teachers already have some knowledge and expertise in this area. However, in other disciplines, e.g. astrology, chemistry and medicine, where virtual laboratories are also very useful, this problem makes it hard to involve teachers in creating new virtual experiments.

Recently it is a subject of active research to fill the gap between this two categories [11] [12]. Therefore, these are still only suitable to a specific set of experiments or rely on software components that were not designed for the given purpose.

\subsection{A New Perspective on Virtual Laboratories}

In order to ensure the usability of different virtual laboratories, members of the scientific community - who are also users of these systems - conducted assessment, most of the time focusing either on the didactic aspects, i.e. how the usage of virtual laboratory increased student's grades or on the implementation aspects, i.e. the used software components. The most common assessment method involves surveying teachers and students on subjective indicators and processing the accumulated data with statistical methods. In a number of these articles, some assumptions can be found on which characteristics of a virtual laboratory are the most influential on the didactic success of the laboratory. However, there is no effort to evaluate these systems from the synergic point of cognitive infocommunication [13] [14]. 
In order to have a better picture on what makes a virtual laboratory system successful, it is necessary to link the cognitive process of learning and the virtual laboratory as an infocommunication system together. This can be done by evaluating virtual laboratories from the point of view of the participants, i.e. the communicating parties. This can help identify the key factors that must be taken into account when designing a new virtual laboratory system.

The aim of a traditional laboratory course is to complement theoretical knowledge, gained through lectures. The participating parties are the institution, i.e. the university, the teacher or laboratory supervisor and the student.

The virtual laboratory can be described as an infocommunication system, which is used by teachers to create virtual experiments and learning materials and by students to gain knowledge through these virtual experiments.

In the virtual laboratory, the teacher or supervisor is not present during the experiment; instead the necessary guidance is presented in the experiment description. Teachers create these descriptions and additional help materials to give the narrative during the virtual experiment. In this way, many students can do the same experiment in the same time, in a self-paced manner.

The aspects of evaluation, described in previous works [1] [2] [15] can be extended with the cognitive aspect as the following.

Aspects of the evaluation from the point of the university and the teachers:

- Technical: How hard is the installation of the virtual laboratory system? How complicated is the process to implement a new experiment? Which software tools or programming languages are supported to implement the simulation?

- Economical: what are the development and operation costs of the virtual laboratory system?

- Cognitive: is a virtual lab suitable for the current topic or concept? Is the user interface consistent and easy-to-use? Is it possible to present all related materials, like help docs and textbooks on a single user interface?

Aspects of the evaluation from the point of view of the student:

- Technical: what are the system requirements of the virtual lab? How complicated is it to access the virtual lab? Is it necessary to install new software?

- Cognitive: Is the virtual laboratory an effective tool to learn? How hard is it to gather all the related information to do the experiment? Is the provided narrative in the laboratory self-contained, and adequate to do the experiment? 


\subsection{Evaulation}

In order to give an overview of the current state of virtual laboratories, several implementations were evaluated, based on the new, cognitive aspects. Since many these implementations are based on a similar design, the systems below were selected to illustrate the distinctive features that were identified during the evaluation.

\subsubsection{Weblab Deusto}

The Weblab Deusto [15] project is a joint effort to give access to real laboratory equipment over the internet for research and educational purposes. It can be categorized as is a remote laboratory system.

From the cognitive aspect, the learning materials in Weblab Deusto are presented on a web-based user interface. It is designed in a way that the relevant and related materials are not presented on the same interface as the remote interface of the experiment. Because of this, the description of the remote experiment and other learning materials must be gathered and accessed separately by the users.

\subsubsection{VISIR}

The Virtual Systems in Reality (VISIR) [11] project aimed to establish a remote laboratory for remote wiring and measurement of electronic circuits on breadboard.

The system design of this remote laboratory follows a modular approach. The measurement equipment used for the experiment is connected to a laboratory server, that is connected to a webserver.

The user interface is web-based and has similar features as a Learning Management system. This allows tutors to create courses, experiment descriptions and additional materials for the experiment with a familiar toolset. However, the experiment itself needs to be implemented by hand, with a specific toolset, using a separate user interface.

\subsubsection{VEMA}

Virtual Electric Manual or VEMA [5] is a Virtual Learning Environment, designed with focus on a high level of realism, in order to create an immersive experience.

From the cognitive aspect, the resemblance between real measurement equipment and the 3 dimensional model representation in the virtual environment helps students to connect the knowledge gained through the virtual experiment to the real world. This approach is promising; however a great amount of manual labor is required to create these high fidelity $3 \mathrm{D}$ models. 


\subsubsection{LDH vLab}

This virtual laboratory aims to implement a web-based lesson to teach lactate dehydrogenase (LDH) enzyme kinetics to 2nd-year biochemistry students [6].

The virtual laboratory is accessible through a web-based user interface. This interface is designed in a way that the description of the experiment and related materials are presented side-by-side with the experiment itself. This reduces the amount of context switching that are necessary to finish the experiment. As described in [6], the overall experience of students was positive, but some of them experienced technical issues which perplexed the learning process.

\section{Design of a New Virtual Laboratory Environment}

\subsection{Frontend}

Looking at existing virtual laboratory implementations [1] [16] and considering the disadvantages of virtual laboratories, it is apparent that there is no consistency in the presentation layer, i.e. in user interfaces. From this problem, the first requirement can be defined as the following; the main user interface, including the administration interface for teachers and the user interface for students must be consistent and intuitive.

The second requirement, which comes from the cognitive aspect is to minimize the necessary context switches during the experiment, i.e. present all relevant and related material on a single user interface.

Nowadays the majority of students mainly use smartphones and tablets to gain access to web services instead of desktop and laptop computers, it is necessary to support these devices.

\subsubsection{Web-based Client}

In order to fulfill this requirement the current best practices from the field of web user interface design should be utilized. As [15] points out, many software components can be used. Therefore, it is advised to avoid technologies like Flash and Java, as the majority of mainstream web services do nowadays because of their many practical problems, like security and the lack of cross-platform operation.

The proposed web-based client interface should use modern web technologies like HTML5 and JavaScript [5] [12] [17]. This way it can be ensured that the user interface can be used in any modern web browser, which is already installed on 
end-user devices like smartphones and tablets. This also ensures that on desktop PCs, the virtual laboratory can be used without the need to install additional software [15].

This approach also enables the usage of modern frameworks, which helps create an intuitive, self-explanatory interface because most of the UI components can be found in other popular web applications, which students already use on a daily basis [18].

\subsubsection{D Client Application}

The proposed desktop client for the virtual laboratory system is based on the MaxWhere [19] framework. MaxWhere is a unique VR framework (engine) that is programmable through JavaScript and allows for building 3D, VR applications in which the conventional web content is fully associative with the $3 \mathrm{D}$, VR world. MAXWHERE provides a JS API called WOM (Where Object Model) that implements similar functionality for VR as the DOM does in web browsers.

The ability to manipulate the models of the 3D space from the programming environment makes it easy to create high fidelity, interactive 3D models which can help students to understand complex 3D structures more easily [20].

The ability to load webpages inside the 3D environment makes MaxWhere a good choice to build upon because the same web-based user interface of the system can be used in the desktop client. This reduces the development effort and also ensures that user interfaces are consistent across the web and desktop clients. Another advantage of this ability is that by using multiple web browser windows placed in a 3D environment, all relevant content - e.g. lecture notes and description of the experiment - can be presented to the user in a single space [21] [22]. Students can also use these web browsers to open relevant and useful web-based products, e.g. their webmail client, online video materials and the university LMS website in this environment [23]. This highly reduces the amount of context switches, i.e. changing between different windows to complete a virtual experiment and helps students to focus on learning.

\subsection{Backend}

The recent trend in large-scale web-based software development and operation is to change the architecture from a monolithic service to microservices [24]. This trend induced the development of new tools to cover all the operation tasks from deployment to management, backup and monitoring. Nowadays the microservice ecosystem reached a maturity level that is considered ready for day-to-day business operation. Therefore, the new system should be composed of a set of modular microservices. This way it is straightforward to create scalable virtual laboratories and all the necessary tools are available to operate it. 
Figure 1 shows the general overview of the proposed new architecture.

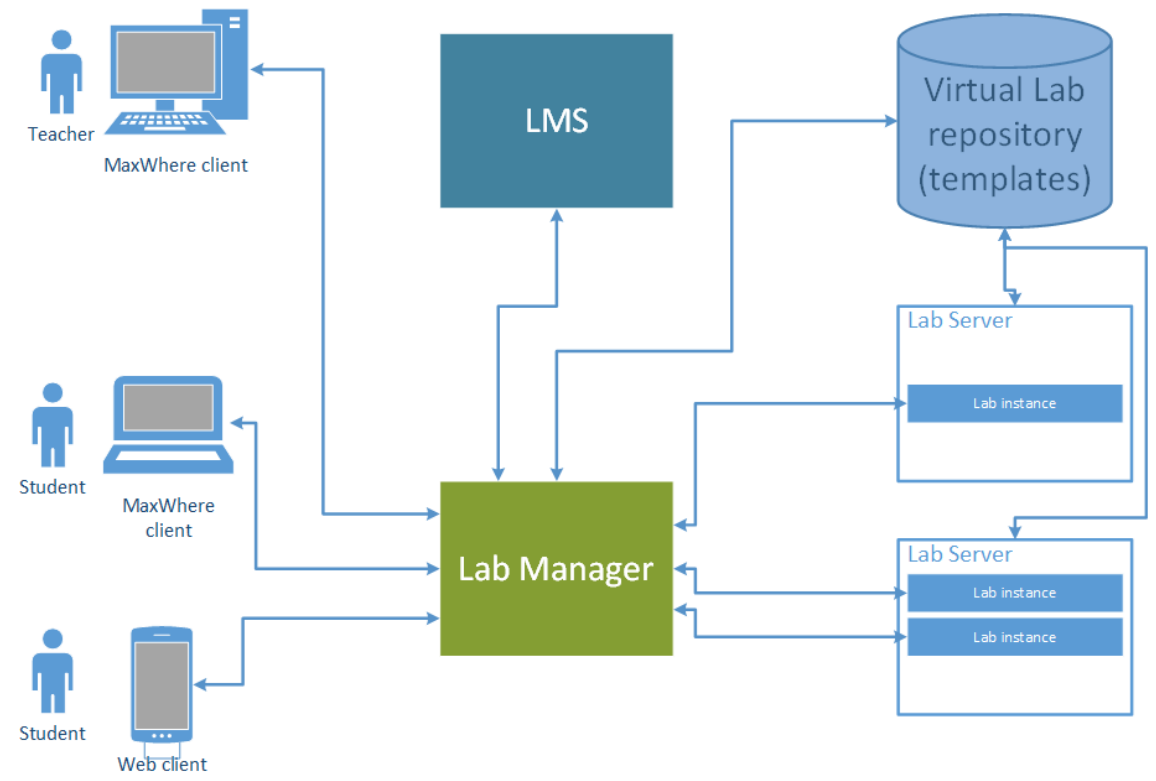

Figure 1

High level architecture of the virtual laboratory framework

\subsubsection{Laboratory Manager}

The core component of the new design is the Laboratory manager. It should provide the web server service, to serve the web application to clients. It should handle the authentication and authorization of clients with the help of the Learning Management System of the university, and it should handle the deployment, management and monitoring of the virtual laboratory instances.

Each of these roles require complex functionality, which means that the Laboratory Manager should be modular, as mentioned earlier, in order to scale well with the increasing number of concurrent users.

\subsubsection{Virtual Lab Repository}

The Virtual Lab repository stores all necessary assets of a virtual laboratory, like textual descriptions, source code or input files of the simulation and virtual laboratory templates. A virtual laboratory template should contain all the properties of a given laboratory, requirements of the simulation in terms of software components and system resources, and a recipe which describes the deployment and execution of the simulation. 
This way, teachers can create different laboratories in a very rapid way, because several assets can be re-used for multiple laboratories and different learning narratives can be defined in the same virtual space.

The Laboratory Manager uses these templates to create, deploy and execute virtual laboratory instances.

\subsection{Integration with Simulation Software Tools}

The main part of a virtual laboratory is the simulation that calculates the actual state of the virtual environment. Therefore, in order to use a virtual laboratory one must run this simulation somewhere. There are two major problems regarding simulation that must be taken into account. First, as the complexity of the laboratory exercise rises, the simulation can quickly get very resource heavy in terms of CPU and memory. Second, these simulations are often implemented using different software packages and libraries, with very different system requirements [25], like the type and version of the operating system.

\subsubsection{Local and Online Operation Modes}

The straightforward solution for the first problem would be to raise the system requirements of virtual laboratory. However, this procedure would effectively shut out a large number of students from the virtual laboratory. In order to solve this problem, the system should be designed following the client-server model. In this way, the virtual laboratory can operate in two different ways based on the system resource requirement of a given simulation.

First, simulations with low resource requirements can be executed on the local machine and the calculated data is then transmitted to the client via a local interface without the need of a network connection. It is important to note that in this mode all software components must be run on the client machine; therefore, it is only suitable to a reduced set of simulation software. This operation mode is called local mode.

Second, simulations with high resource requirements can be executed on a remote server [26] and the calculated data is then transmitted to the client via the network. This operation mode is called online mode.

\subsubsection{Standard Simulation Software Interface}

The solution for the second problem would be to set up a list of simulation software and libraries which the teacher can choose from when implementing the virtual experiment. However, in this case the teacher is required to re-implement simulations that are already implemented in software that is not part of the list. 
Instead, the new system should establish a new interface to exchange input and output data with the simulation software. This can be used to create wrappers for different simulators.

\subsection{Integration with Learning Management Systems}

The new system should be able to be integrated with widely used Learning Management Systems, like Moodle [15] [27]. The Laboratory Manager should use the LMS to authenticate and authorize teachers and students. It should be able to logically connect virtual laboratory exercises, defined by the teacher in the LMS and in the Manager. This way, the different interactions in a virtual laboratory can be defined as course requirements in the LMS. For example, the completion if a virtual laboratory can be set up as an obligatory requirement to pass a given course. In order to fulfill this requirement, the Manager should be able to collect and $\log$ the interactions of students in virtual laboratories. This can be achieved by using existing web monitoring and logging tools [28].

\section{Conclusions}

The new perspective of using cognitive infocommunication to describe the learning process in a virtual laboratory helped to pinpoint new important factors that a creator of a virtual laboratory system should take into account, in order to achieve a high didactic potential. The described requirements of the new virtual laboratory system can function as a design pattern for new implementations.

The next step is to implement a new virtual laboratory system, based on this design. This new system then will be tested with the help of tutors and students in order to validate the design and to ensure that the new system successfully fulfills all the described requirements in this paper.

\section{Acknowledgement}

This work was supported by the FIEK program (Center for cooperation between higher education and the industries at the Széchenyi István University, GINOP2.3.4-15-2016-00003)

\section{References}

[1] V. Potkonjak, M. Gardner, V. Callaghan, P. Mattila, C. Guetl, V. M. Petrović, K. Jovanović. Virtual laboratories for education in science, technology, and engineering: A review. Computers \& Education, Vol. 95, 2016, pp. 309-327

[2] Advances on remote laboratories and e-learning experiences. Universidad de Deusto, 2008, ISBN: 9788498306620

[3] M. Auer, A. Pester, D. Ursutiu, C. Samoila. Distributed virtual and remote labs in engineering. IEEE International Conference on Industrial Technology, 2003 
[4] A. Peidró, O. Reinoso, A. Gil, J. M. Marín, L. Payá. A virtual laboratory to simulate the control of parallel robots, IFAC-PapersOnLine, Vol. 48 No. 29, 2015, pp. 19-24

[5] M. Valdez,C. M. Ferreira, F. P. M. Barbosa. 3D Virtual Laboratory for Teaching Circuit Theory - A Virtual Learning Environment (VLE) $51^{\text {st }}$ International Universities' Power Engineering Conference, 2016 DOI:10.1109/UPEC.2016.8114126

[6] C. Booth, R. Cheluvappa, Z. Bellinson, D. Maguire, C. Zimitat, J. Abraham, R. Eri. Empirical evaluation of a virtual laboratory approach to teach lactate dehydrogenase enzyme kinetics, Annals of Medicine and Surgery, Vol. 8, 2016, pp. 6-13, ISSN 2049-0801

[7] M. de Massimilian. A MATLAB-based virtual laboratory for teaching introductory quasi-stationary electromagnetics. IEEE Transactions on Education Vol. 48, No. 1, 2005, pp. 81-88

[8] A. Ahmad, M. K. Nordin, M. F. Saaid, J. Johari, R. A. Kassim and Y. Zakaria, Remote control temperature chamber for virtual laboratory, 2017 IEEE $9^{\text {th }}$ International Conference on Engineering Education (ICEED) Kanazawa, 2017, pp. 206-211

[9] M. B. Erdem, A. Kiraz, H. Eski, Ö. Çiftçi, C. Kubat. A conceptual framework for cloud-based integration of Virtual laboratories as a multiagent system approach. Computers \& Industrial Engineering, Vol. 102, 2016, pp. 452-457

[10] H. Majid, H. F. Manesh, M. Bal. A modular virtual reality system for engineering laboratory education. Computer Applications in Engineering Education Vol. 19, No. 2, 2011, pp. 305-314

[11] T. Mohamed, et al. Virtual instrument systems in reality (VISIR) for remote wiring and measurement of electronic circuits on breadboard, IEEE Transactions on Learning Technologies Vol. 6, No. 1, 2013, pp. 60-72

[12] F. Esquembre. Facilitating the Creation of Virtual and Remote Laboratories for Science and Engineering Education. IFAC-PapersOnLine, Vol 48, No 29, 2015, pp. 49-58, ISSN 2405-8963

[13] P. Baranyi, A. Csapo. Definition and Synergies of Cognitive Infocommunications, Acta Polytechnica Hungarica, Vol. 9, No. 1, 2012, pp. 67-83

[14] P. Baranyi., A. Csapo, G. Sallai. Cognitive Infocommunications (CogInfoCom). Springer International Publishing, 208 pages, 2015, ISBN: 978-3-319-19607-7

[15] J. Garcia-Zubia, P. Orduna, D. Lopez-de-Ipina, G. R. Alves. Addressing software impact in the design of remote laboratories. IEEE Transactions on Industrial Electronics, Vol. 56, No. 12, 2009, pp. 4757-4767 
[16] P. Trnka, S. Vrána, B. Šulc, Comparison of Various Technologies Used in a Virtual Laboratory, IFAC-PapersOnLine, Vol. 49, No. 6, 2016, pp. 144-149

[17] HTML5 specification, https://www.w3.org/TR/html5/ [online, last visited on: 2018.01.04]

[18] I. Horváth: Digital Life Gap between students and lecturers. In: Proceedings of $7^{\text {th }}$ IEEE Conference on Cognitive Infocommunications. Wrocław, Poland, 16-18.10.2016. Budapest: IEEE Hungary Section, 2016, pp. $353-358$

[19] MaxWhere website, http://www.maxwhere.com/ [online, last visited on: 2018.01.04]

[20] Z. Kvasznicza: Teaching electrical machines in a 3D virtual space, $8^{\text {th }}$ IEEE International Conference on Cognitive Infocommunications, Debrecen, 2017

[21] G. Csapó, Sprego Virtual Collaboration Space, $8^{\text {th }}$ IEEE International Conference on Cognitive Infocommunications, 2017

[22] I. Horváth: Disruptive technologies in higher education, In: Proceedings of $7^{\text {th }}$ IEEE Conference on Cognitive Infocommunications, Wrocław, Poland, 16-18.10.2016. Budapest: IEEE Hungary Section, 2016, pp. 347-352 (ISBN 978-1-5090-2644-9; 978-150902645-6)

[23] I. Horváth: Innovative engineering education in the cooperative VR environment, In: Proceedings of $7^{\text {th }}$ IEEE Conference on Cognitive Infocommunications. 496 p. Wrocław, Poland, 16-18.10.2016. Budapest: IEEE Hungary Section, 2016, pp. 359-364 (ISBN 978-1-5090-2644-9; 978150902645-6)

[24] M. Villamizar et al., Evaluating the monolithic and the microservice architecture pattern to deploy web applications in the cloud, $201510^{\text {th }}$ Computing Colombian Conference (10CCC) Bogota, 2015, pp. 583-590

[25] I. Horváth: The IT device demand of edu-coaching in the higher education of engineering, In: IEEE $8^{\text {th }}$ International Conference on Cognitive InfoCommunications: CogInfoCom. Debrecen, Hungary, 11-14.09.2017. (IEEE) Piscataway: IEEE Computer Society, 2017, pp. 379-384

[26] S. D. Burd, X. Luo, A. F. Seazzu. Cloud-based virtual computing laboratories. $46^{\text {th }}$ Hawaii International Conference on System Sciences, 2013, pp. 5079-5088, IEEE

[27] M. Dougiamas, P. Taylor, Moodle: Using Learning Communities to Create an Open Source Course Management System. In: World Conference on Educational Multimedia, Hypermedia and Telecommunications (EDMEDIA) 2003, Chesapeake, VA, USA

[28] R. Atterer, M. Wnuk, A. Schmidt. Knowing the user's every move: user activity tracking for website usability evaluation and implicit interaction. In Proceedings of the $15^{\text {th }}$ international conference on World Wide Web (WWW '06) ACM, New York, NY, USA, 2006 pp. 203-212 